\title{
GENETIC SLOW-MODE/FAST-MODE OPTIMISATION OF DIGITAL PID CONTROLLERS
}

\author{
B Porter and D L Hicks \\ Department of Aeronautical, Mechanical, and Manufacturing Engineering \\ University of Salford \\ Salford M5 4WT \\ England
}

\begin{abstract}
The optimal parameter settings for digital PID controllers incorporated in model-following systems are complicated, unknown functions of the underlying sampling frequency. In order to simplify the design process, genetic algorithms have previously been used to tune the restricted set of controller parameters affecting the fast modes of the closed-loop system. However, it is important to investigate the effects of both slow and fast modes on model-following behaviour by using an enlarged set of controller parameturs in the genetic optimisation procedure. This use of genetic algorithms is illustrated in this paper by the design of model-following flight-control systems for the F-16 aircraft.
\end{abstract}

\section{INTRODUCTION}

The methodologies of Porter et al [1] provide a powerful means of designing model-following systems incorporating digital PID controllers. These methodologies use only plant input/output data and thus circumvent the need for explicit mathematical models of multivariable plants. Indeed, it was shown by Porter [2] that such model-following systems can be designed simply by using the step-response matrices of multivariable plants. Since such step-response matrices can be directly identified in real time using standard recursive parameter-estimation techniques, such model-following systems can readily be rendered adaptive.

However, in order to obtain optimal performance it is necessary to tune the digital PID controllers in such non-adaptive or adaptive model-following systems. The underlying singular perturbation theory developed by Porter et al [1][7] provides asymptotically optimal parameter settings for such PID controllers as the associated sampling frequencies become very large. However, in practice, such sampling frequencies are finite and the resulting non-asymptotically optimal controller parameter settings may differ significantly from their asymptotically optimal settings. This use of genetic algorithms for tuning such digital PID controllers was therefore investigated by Porter and Hicks [4][5][6].

The results obtained from the genetic design procedure [4][5][6] provide improved model-following behaviour compared with that obtained using the asymptotic design procedure [1]. However, in the genetic optimisation procedure of Porter and Hicks [4][5][6], only the restricted set of controller parameters affecting the fast modes of the closed-loop system was used. Since the slow modes of the model-following systems are only asymptotically uncontrollable or unobservable, such slow modes will be present to some extent in the output of model-following systems for finite sampling frequencies. It is therefore important to investigate the effects of both slow and fast modes on model-following behaviour by using an enlarged set of controller parameters in the genetic optimisation procedure.

This use of genetic algorithms is illustrated in this paper by the design of model-following flight-control systems for the F-16 aircraft.

\section{GENETIC DESIGN PROCEDURE}

The closed-loop digital model-following systems under investigation incorporate the following two principal components, as shown in Figure 1:

(i) an explicit multivariable dynamical model that generates desired model output vectors, $w(t)$, in response to command input vectors, $v(t)$;

(ii) a multivariable digital PID controller that generates appropriate control input vectors, $u(t)$, in response to errors between model output vectors, $w(t)$, and plant output vectors, $y(t)$. 


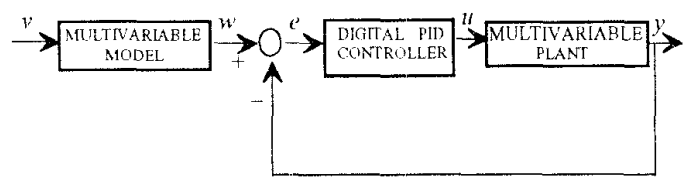

Figure 1: Block diagram of digital model-following system.

It is assumed that the linear multivariable plants under consideration are governed on the continuous-time set $T=[0,+\infty)$ by state and output equations of the respective forms

$$
\dot{x}(t)=A x(t)+B u(t)
$$

and

$$
y(t)=C x(t)
$$

In addition, it is assumed that the fast-sampling digital PID controllers are governed on the discrete-time set $T_{\mathrm{T}}=\{0, T, 2 T, \ldots, k T, \ldots\}$ by control-law equations of the form

$$
u(k T)=K_{1}(T) r(k T)+K_{2}(T) z(k T)
$$

where $T \in R^{+}$is the sampling period. Such controllers are required to generate the piecewise-constant control input vector $u(t)=u(k T), t \in[k T,(k+1) T), k T \in T_{T}$, so as to cause the plant output vector, $y(t)$, to track the model output vector, $w(t)$, on $T_{T}$. This means that the error vector $e(t)=w(t)-y(t)$ is required to assume the steady-state value

$$
\lim _{k \rightarrow \infty} e(k T)=\lim _{k \rightarrow \infty}\{w(k T)-y(k T)\}=0
$$

on $T_{T}$ for arbitrary initial conditions.

In equations (1), (2), (3), and (4), $x(t) \in R^{n}, u(t) \in R^{\prime}$, $y(t) \in R^{l}, e(t) \in R^{l}, w(t) \in R^{l}, A \in R^{n x m}, B \in R^{n x l}, C \in R^{l x n}$, $z(k T) \in R^{l}, r(k T) \in R^{l}, K_{1}(T) \in R^{l x l}, K_{2}(T) \in R^{l x l}, T \in R^{+}$,

and

$$
\operatorname{rank} C B=i-p
$$

The plant outputs are ordered such that

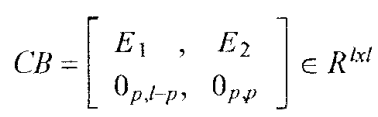

where $E_{1} \in R^{(l-p) x(l-p)}, E_{2} \in R^{(l-p) x p}$, rank $E_{1}=l-p$, and the non-negative integer $p$ is the rank defect of the first Markov parameter of the open-loop plant. Furthermore, the vectors $r(k T)$ and $z(k T)$ in equation (3) are generated in accordance with the difference equations

$$
s\{(k+1) T\}=-\alpha I_{p} s(k T)+\left[0_{p, t-p}, I_{p}\right] e(k T),
$$

$$
r(k T)=-\left[\begin{array}{c}
\frac{2}{T}(1+\alpha) D_{2} \\
\frac{2}{T}(1+\alpha) D_{4}
\end{array}\right] s(k T)+\left(I_{l}+\frac{2}{T} D\right) e(k T),
$$

and

$$
z\{(k+1) T\}=z(k T)+\operatorname{Tr}(k T)
$$

In equations (7), (8), and (9), $\alpha \in(-1,+1), s(k T) \in R^{p}$, and the derivative matrix

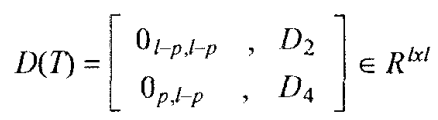

is such that

$$
D(T) C B=0_{l, l}
$$

where $D_{2}(T) \in R^{(l-p) x p}$ and $D_{4}(T) \in R^{p x p}$.

The frequency-domain characteristics of such closed-loop digital model-following systems can be conveniently elucidated by determining for such systems the closed-loop transfer function matrix, $G(z)$, relating the plant output vector to the desired model output vector. The results of Porter and Shenton [7] from the singular perturbation analysis of transfer function matrices can then be used to obtain the asymptotic form of $G(z)$ as the sampling frequency $f=1 / T \rightarrow \infty$. These results thus greatly facilitate the determination of the controller matrices $K_{l}(T) \in R^{i x l}$ and $K_{2}(T) \in R^{l x l}$, together with the derivative matrices $D_{2}(T) \in R^{(l-p) x p}$ and $D_{4}(T) \in R^{p x p}$, such that the resulting fast-sampling digital PID controller produces closed-loop model-following behaviour of increasing fidelity as the sampling frequency is increased. Indeed, in terms of the open-loop step-response matrix

$$
H(T)=\int_{0}^{T} C e^{A t} B d t \in R^{l x l},
$$

and the associated regularity matrix 


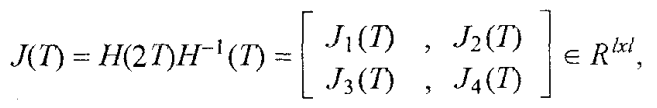

this singular perturbation analysis indicates that the appropriate design equations for the digital PID controller are

$$
\begin{aligned}
& K_{1}(T)=T H^{-1}(T) \sum\left(T I_{l}+2 D\right)^{-1}, \\
& K_{2}(T)=\rho K_{1}(T),
\end{aligned}
$$

and

$$
(1+\alpha) \Sigma_{1} D_{2}(T)=J_{2}(T) \Sigma_{2} D_{4}(T)
$$

where

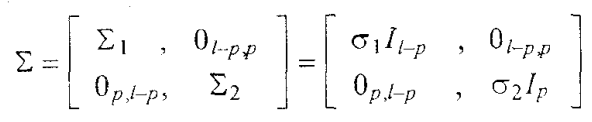

and $\sigma_{1}, \sigma_{2}, \rho, \delta_{4}(T) \in R^{+}$. The closed-loop transfer function matrix, $G(z)$, then assumes the diagonal asymptotic form

$$
\Gamma(z)=\left[\begin{array}{ccc}
\frac{\sigma_{1}}{z-1+\sigma_{1}} I_{l-p} & , & 0_{l-p p} \\
0_{p, l-p} & , & \frac{\sigma_{2}(z+1)}{z^{2}+\left(\alpha+\sigma_{2}-1\right) z+\sigma_{2}-\alpha} I_{p}
\end{array}\right]
$$

as $f=\frac{1}{T} \rightarrow \infty$, thus indicating that fast and non-interacting model-following behaviour occurs.

This closed-loop model-following behaviour is a consequence of the facts [1] that the slow modes of the resulting closed-loop system become either asymptotically unobservable or uncontrollable as $f=\frac{1}{T} \rightarrow \infty$ and that the fast modes of the closed-loop system remain both controllable and observable. Hence, only the fast modes (which depend only on the restricted set of controller parameters $\left.\left\{\sigma_{1}, \sigma_{2}, \alpha\right\}\right)$ appear in the asymptotic closed-loop transfer-function matrix as $f=\frac{1}{T} \rightarrow \infty$.

Indeed, the singular perturbation analysis [1][7] leading to the asymptotic transfer-function matrix, $\Gamma(z)$, in equation (18) indicates that the optimal choice of design parameters is $\left\{\sigma_{1}, \sigma_{2}, \alpha\right\}=\left\{1, \frac{1}{2}, \frac{1}{2}\right\}$ since it is then evident that all the poles of the resulting asymptotic transfer-function matrix lie at the origin within the unit disc. However, in practice, the sampling frequencies of fast-sampling digital PID controllers are obviously finite and the associated optimal parameter settings mat well differ significantly from their asymptotically optimal settings.

Genetic algorithms have previously been used $[4][5][6]$ to tune the restricted set of controller parameters $\left\{\sigma_{1}, \sigma_{2}, \alpha\right\}$ for finite sampling frequencies. This restricted set facilitates the optimisation of the fast modes of the closed-loop system whilst the remaining controller parameters (which essentially determine the slow modes of the system) are assigned fixed values (for example, $\rho=1$ and $\delta=0.25$ ).

However, since the slow modes of the closed-loop system are present to some extent in the outputs of the closed-loop system for finite sampling frequencies, it is therefore pertinent to investigate the effects of slow-mode/fast-mode tuning on model-following behaviour by using the enlarged controller parameter set $\left\{\sigma_{1}, \sigma_{2}, \alpha, \rho, \delta\right\}$ for such finite sampling frequencies.

Thus, for example, if minimum maximum multivariable generalised model-following error is regarded as the ultimate design requirement, genetic algorithms can be readily used to select the enlarged set of controller parameters such that the measure of generalised model-following error

$$
\varepsilon=\sum_{i=1 j=1}^{1} \sum_{j=1}^{1}\left\{\omega_{i j} \max _{t}\left|e_{j}^{(i)}(t)\right|+\sum_{i=1 j=1}^{l} \sum_{j=1}^{1}\left\{\mu_{i j} \max _{r, t(i m p u l s e)}\left|\Delta u_{j}^{(i)}(t)\right|\right\}\right.
$$

is minimised. In this measure of generalised model-following error, $e_{j}^{(i)}(t)$ is the model-following error in the $j t h$ channel when a command is applied to the $i t h$ channel, $\Delta t_{j}^{(i)}(t)$ is the corresponding change in the $j t h$ control input (over a sampling period), and $\omega_{i j}$ and $\mu_{i j}$ are weighting parameters.

\section{ILLUSTRATIVE EXAMPLE}

This procedure for the genetic slow-mode/fastmode design of digital model-following systems for finite sampling frequencies can be conveniently illustrated by considering the F-16 aircraft [1] for which a digital PID controller was previously designed both non-genetically [1] and genetically [4] using the restricted controller parameter set.

It is desired to design a digital PID controller that minimises the maximum multivariable generalised model-following crror when the F-16 aircraft performs pitch-pointing and vertical-translation manoeuvres for the F-16 flying at Mach 0.9 at an altitude of $15,000 \mathrm{ft}$. In these manoeuvres, it is known that practical position and rate limits [8] are 
comfortably satisfied by selecting models with transfer function [1]

$$
g(s)=\frac{50}{(s+2)\left(s^{2}+8 s+25\right)} .
$$

in both the pitch-angle and flight-path-angle channels.

The results of solving this model-following design problem for the enlarged controller parameter set (with $\omega_{i j}=1$ and $\mu_{i j}$ $=0.01$ in equation (19)) by means of a genetic algorithm with a population size $\mathrm{N}=50$, a crossover probability $p_{c}=0.6$, and a mutation probability $p_{m}=0.01$ are shown in Figure 2 over 100 generations. In Figure 2(a) the best-of-generation measure of generalised model-following error is plotted against generation number whilst, in Figures 2(b), (c), (d), (e), and $(f)$, the associated best-of-generation controller parameters $\sigma_{1}, \sigma_{2}, \alpha, \rho$, and $\delta$ are respectively plotted against generation number. It can be seen from Figures 2(a), (b), (c), and (d) that the minimal generalised model-following error $\varepsilon=0.0272$ and that the associated optimal enlarged controller parameter set is $\left\{\sigma_{1}, \sigma_{2}, \alpha, \rho, \delta\right\}=$ $\{1.119,0.641,0.5423,13.728,0.2597\}$. The time-domain model-following behaviour corresponding to this set of optimal controller parameters is shown in Figure 3. It is evident that the slow-mode/fast-mode genetically tuned digital PID controller gives excellent model-following behaviour. Indeed, this behaviour is superior to that obtained in the case of the fast-mode genetically tuned digital PID controller for which [9] the minimal value of the generalised model-following error $\varepsilon=0.0621$ with the associated optimal restricted controller parameter set $\left\{\sigma_{1}, \sigma_{2}, \alpha\right\}=$ $\{1.081,0.4915,0.4801\}$ (with $\rho=1$ and $\delta=0.25$ ).

\section{CONCLUSION}

In this paper, genetic algorithms have been used to tune the enlarged controller parameter set $\left\{\sigma_{1}, \sigma_{2}, \alpha, \rho, \delta\right\}$ for fast-sampling digital PID controllers for finite sampling frequencies. This enlarged controller parameter set facilitates the tuning of both slow and fast modes of the closed-loop system for finite sampling frequencies, and significant improvements have thus been made in the case of the F-16 aircraft when compared to the results previously obtained non-genetically [1]. This genetic slow-mode/fast-mode optimisation procedure has also been shown to lead to improved model-following behaviour when compared to the results previously obtained from genetic fast-mode optimisation [9].

\section{REFERENCES}

1. B Porter, A Manganas, and T Manganas, "Design of digital model-following flight-mode control systems for high-performance aircraft", Proc AIAA Guidance, Navigation and Control Conference, Minneapolis, USA, August 1988.

2. B Porter, "Design of set-point tracking systems incorporating fast-sampling error-actuated digital controllers for linear multivariable plants using step-response matrices", Proc IFAC World Congress, Munich, Germany, July 1987.

3. B Porter and $M Z$ Othman, "Design of adaptive digital model-following flight-mode control systems for high-performance aircraft", Proc AIAA Guidance, Navigation and Control Conference. Boston, USA, August 1989.

4. B Porter and D L Hicks, "Genetic design of digital model-following flight-control systems", Proc AIAA Guidance, Navigation and Control Conference, Monterey, USA, August 1993.

5. B Porter and D L Hicks, "Performance measures in the genetic design of digital model-following flight-control systems", Proc IEEE National Aerospace and Electronics Conference, Dayton, USA, May 1994.

6. B Porter and D L Hicks, "Genetic robustification of digital model-following flight-control systems", Proc IEEE National Aerospace and Electronics Conference, Dayton, USA, May 1994.

7. B Porter and A T Shenton, "Singular perturbation analysis of the transfer function matrices of multivariable linear systems", Int J Control, Vol 21, pp 655-660, 1975.

8. A F Barfield and J J D'Azzo, "Multivariable control laws for the ATFI/F-16", Proc AIAA 22nd Aerospace Sciences Meeting, Reno, USA, January 1984.

9. D L Hicks, "Optimal Design of Digital Model-Following Systems", PhD Thesis, University of Salford, Salford, UK, 1994. 


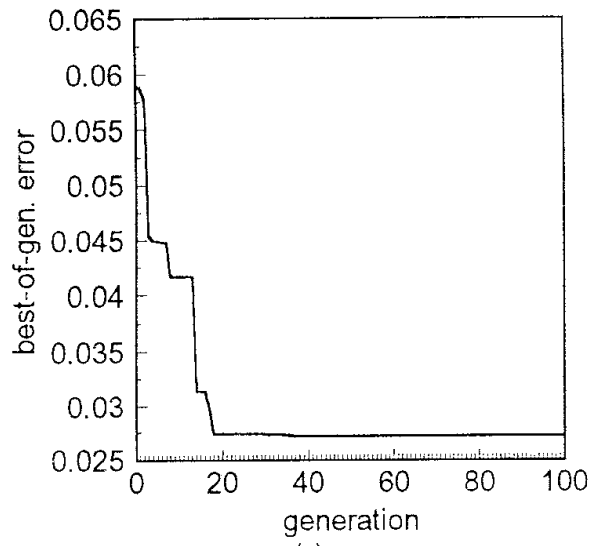

(a)

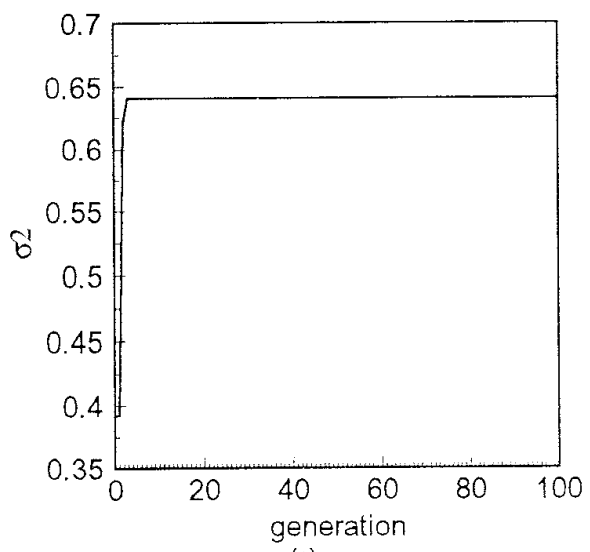

(c)

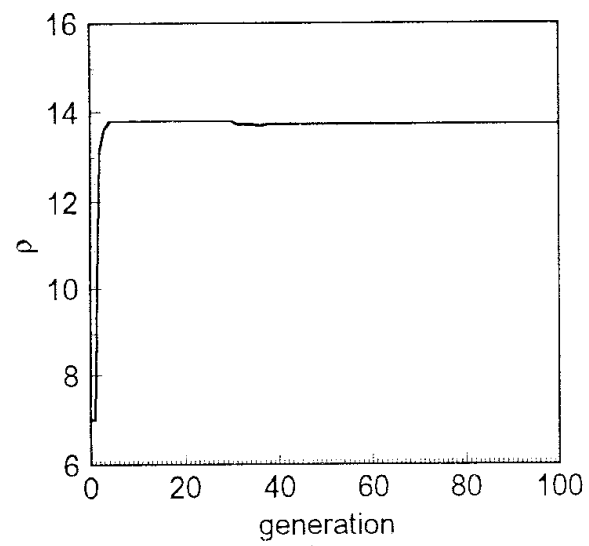

(e)

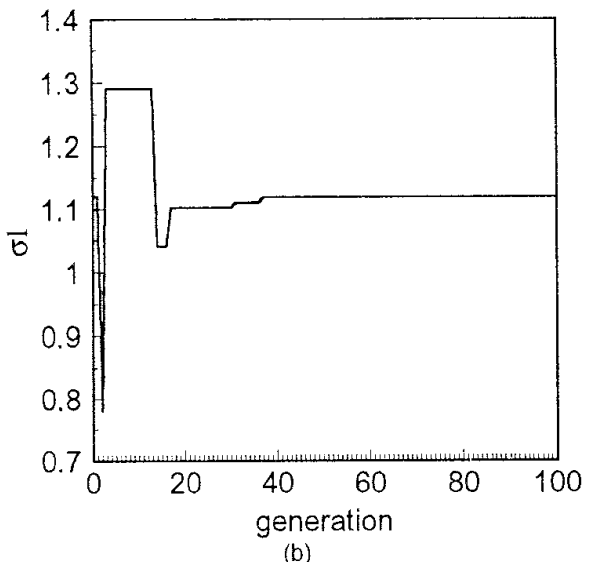

(b)

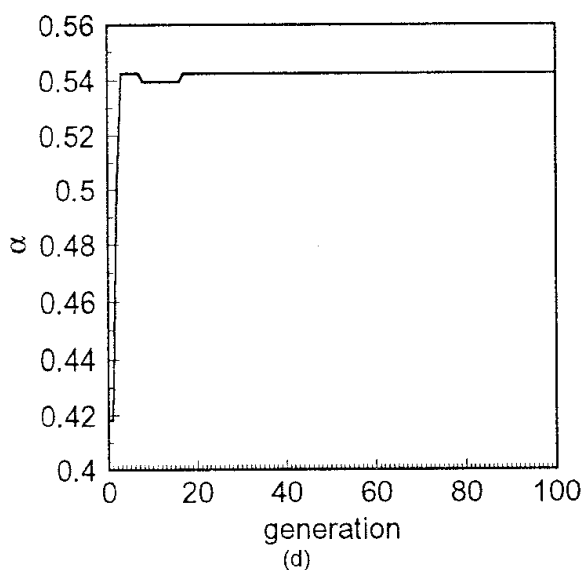

(d)

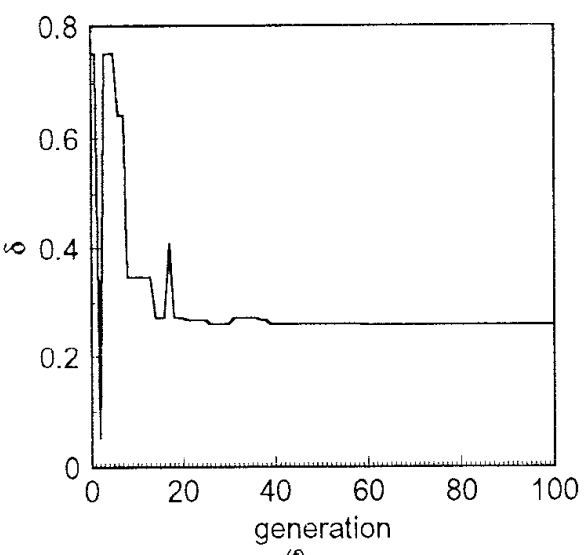

(f)

Figure 2: Best-of-generation generalised model-following error and controller parameters ( $T=0.01)$ 

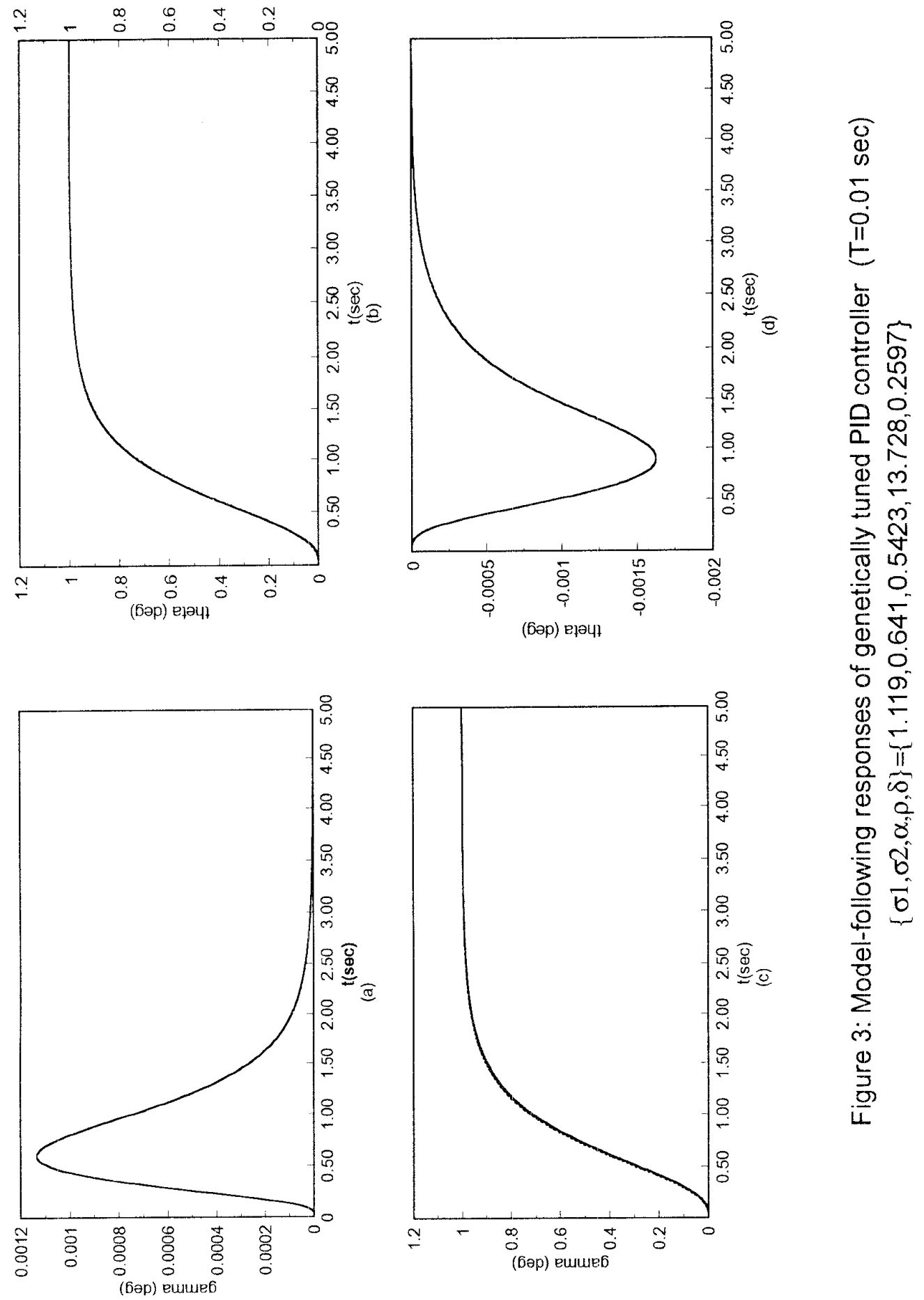